\title{
Interventional Stroke Treatment in Patients with Tandem Lesions: What Factors Influence the Clinical Outcome?
}

\author{
Elmouden $R^{1}$, Moya $A^{1}$, Reimann $G^{2}$, Adamczewski $O^{1}$, Schwarz $M^{2}$ and Rohde $S^{1 *}$ \\ ${ }^{1}$ Radiological and Neuroradiological Department, Klinikum Dortmund gGmbH, Dortmund, Germany \\ ${ }^{2}$ Department of Neurology, Klinikum Dortmund gGmbH, Dortmund, Germany
}

*Corresponding author: Stefan Rohde, MD, Department of Radiology and Neuroradiology, Klinikum Dortmund-Academic Teaching Hospital of the University of Muenster Medical School, Beurhausstrasse 40, 44137 Dortmund, Germany, Tel: +49-231-953-21350, Fax: +49-231-953-21025

\begin{abstract}
Introduction: Endovascular treatment of acute stroke patients with tandem lesions is technically challenging. We sought to evaluate clinical and interventional factors that might influence the clinical outcome in these patients.

Methods: We retrospectively analysed the interventional and clinical data of patients with tandem lesions and acute embolic stroke in the anterior circulation that underwent endovascular stroke treatment at our institution. Patients were selected if they had a CTA-proved vessel occlusion of the distal ICA or M1/2 segment and absence of early signs of advanced infarction (ASPECT score $\geq 6$ ). The primary endpoint of this study was the proportion of patients with good clinical outcome (mRS-score $\leq 2$ ) at 90-day-follow-up. A multivariate analysis (Random forest approach) was used to investigate the effect of several clinical and interventional factors on clinical outcome.

Results: From January 2012 to February 2018, 41 patients (mean age $65.5 \pm 10.2$ years; female, $n=12$ ) with tandem lesions in the anterior circulation (Carotid-T, $n=11$; $M 1$, $n=25 ; M 2, n=5$ ) underwent endovascular treatment at our institution. Stenting of the extracranial ICA and successful recanalization of the occluded vessel segment $(\mathrm{TICl}$ $2 \mathrm{~b} / 3$ ) was possible in all cases. A good clinical outcome at 90 -days-follow-up ( $\mathrm{mRS} \leq 2)$ was documented in 25 of 38 patients $(65.7 \%)$. The overall complication and mortality rate was $10 \%$ and $17 \%$, respectively.

In multivariate testing, a favourable clinical outcome on follow-up correlated with a younger age ( $<70$ years), a more peripheral intracranial vessel occlusion (M1/M2 versus Carotid-T), and good cerebral collaterals $(p<0.05)$. Interventional factors had no statistical effect on clinical outcome.
\end{abstract}

Conclusion: Interventional treatment of acute stroke patients with tandem lesions is technically feasible and safe. A favourable outcome may be achieved in the majority of patients and is most probable in younger patients with peripheral intracranial occlusion and good cerebral collaterals.

\section{Keywords}

Tandem lesions, Endovascular stroke treatment, Carotid stenting, Thrombectomy, Multivariate analysis, Random forest

\section{Introduction}

Since the publication of the results of the randomized trials on mechanical thrombectomy (MT) in 2015, MT has become an established treatment option in the management of acute stroke patients in neuro-vascular centres worldwide [1-5]. In approximately $10 \%-20 \%$ of patients with stroke, additional ipsilateral high-grade ICA stenosis is present [6]. Endovascular treatment patients with tandem lesions is challenging [7-9] as it complicates endovascular access and requires more technical skills than MT for intracranial occlusion alone. Moreover, primary stenting of the extracranial occlusion may lead to a delay in recanalization of the target vessel occlusion. On the other hand, patients with high-grade ICA stenosis might profit from pre-existing intracranial collaterals that-theoretically- can extend the time window for endovascular treatment in terms of beneficial clinical outcome.

The aim of this study was to evaluate different clin-

Citation: Elmouden R, Moya A, Reimann G, Adamczewski O, Schwarz M, et al. (2020) Interventional Stroke Treatment in Patients with Tandem Lesions: What Factors Influence the Clinical Outcome?. Int Arch Vasc Med 3:006. doi.org/10.23937/2690-3164/1710006 Accepted: November 03, 2020: Published: November 05, 2020

Copyright: (c) 2020 Elmouden R, et al. This is an open-access article distributed under the terms of the Creative Commons Attribution License, which permits unrestricted use, distribution, and reproduction in any medium, provided the original author and source are credited. 
ical and interventional factors that may influence the clinical outcome in these patients.

\section{Methods}

\section{Inclusion criteria and patient selection}

The study was approved by the local ethics committees. We retrospectively analysed the interventional and clinical data of patients with acute embolic stroke in the anterior circulation and a cervical high-grade atherosclerotic stenosis or occlusion of the ipsilateral internal carotid artery that underwent endovascular stroke treatment and simultaneous stenting of the ICA at our institution from January 2012 to February 2018.

Patients were selected for endovascular stroke treatment if they had a CTA-proved vessel occlusion of the distal ICA or M1/2 segment and absence of early signs of an extensive and advanced infarction (ASPECT score $\geq 6$ ). Only patients with concomitant atherosclerotic lesions of the extracranial ICA were included into the study; patients with dissections or chronic occlusions were not included.

\section{Interventional procedure}

All procedures were executed by experienced interventional (neuro) radiologists (> $40 \mathrm{MTs} /$ year). The choice of the interventional technique and the devices used was left to the discretion of the interventionist. All procedures were performed under general anaesthesia.

An 8F sheath (Super Arrow-Flex, 8F, Arrow International, Reading, PA, USA) was placed into the affected common carotid artery via a transfemoral access. After exposure of the ICA-bifurcation, the stenosis or occlusion was passed with a 0.014 -inch microwire and pre-dilated with a PTA-balloon to allow fast access to the intracranial vasculature. If passage of the stenosis with an intermediate catheter was possible without immediate stent placement, stenting of the ICA was performed after thrombectomy ("retrograde stenting"). Otherwise, stent implantation was performed prior to cerebral thrombectomy ("antegrade stenting"). Self-expanding stents with closed-cell design were used in all cases (e.g., Wallstent, Xact-Stent, Caspar); additional balloon angioplasty was performed under cardio-protection with atropine.

For intracranial thrombectomy, an intermediate catheter (6F Sophia or Sophia Plus; Microvention, Aliso Viejo, Ca, USA) was advanced through the stent and positioned as close to the thrombus as possible. Mechanical thrombectomy was performed with a stent-retriever (Solitaire, Medtronic, Irvine, Ca, USA) under continuous distal aspiration.

\section{Outcome assessment}

Follow-up CT imaging was performed 24-36 hours after the procedure or whenever clinical worsening occurred. Haemorrhage was classified according to the European Cooperative Acute Stroke Study (ECASS)-2 classifications. Patients were assessed clinically on admission (NIHSS), at discharge (NIHSS, mRS), and on follow-up after 90 days (mRS). Imaging and interventional data were reviewed by two interventionalists (R.E., S.R.).

The primary endpoint of this study was the proportion of patients with good clinical outcome after endovascular stroke treatment, defined by a modified Ranking Scale (mRS)-score $\leq 2$ at 90 day follow-up. Follow-up outcome was assessed during an in hospital visit or by a semi structured telephone interview by a neurologist not blinded to the initial treatment.

\section{Variables and statistical analysis}

To investigate the influence of different factors on the clinical outcome after endovascular treatment, we analysed several clinical and interventional parameters (Table 1). Data were extracted from the prospectively collected stroke database of our institution. Continuous data were collected in a data base and described by median and interquartile range or as mean and SD in case of normal distribution. Differences between quantitative

Table 1: Interventional and clinical factors that were tested for the effect on clinical outcome after endovascular treatment of patients with tandem lesions $(n=41)$.

\begin{tabular}{|l|l|}
\hline Clinical factors & Interventional factors \\
\hline Age, gender & Antegrade/retrograde stenting \\
\hline Co-morbidities & Procedural time \\
\hline Degree of extracranial Stenosis & No. of thrombectomy manoeuvres \\
\hline Site of intracranial vessel occlusion & Successful recanalization (TICl 2b and 3) \\
\hline IV Lysis yes/no & \\
\hline NIHSS on admission & \\
\hline ASPECT score & \\
\hline Collateral status (according to Tan) & \\
\hline Time from symptom onset to recanalization & \\
\hline
\end{tabular}

IV Lysis: Intravenous administration of rtPA; NIHSS: National Institutes of Health Stroke Scale; ASPECT: Alberta Stroke Program Early CT Score; TICl: Thrombolysis in cerebral infarction Score. 
Table 2: Clinical data of 41 patients with tandem lesions of the anterior circulation.

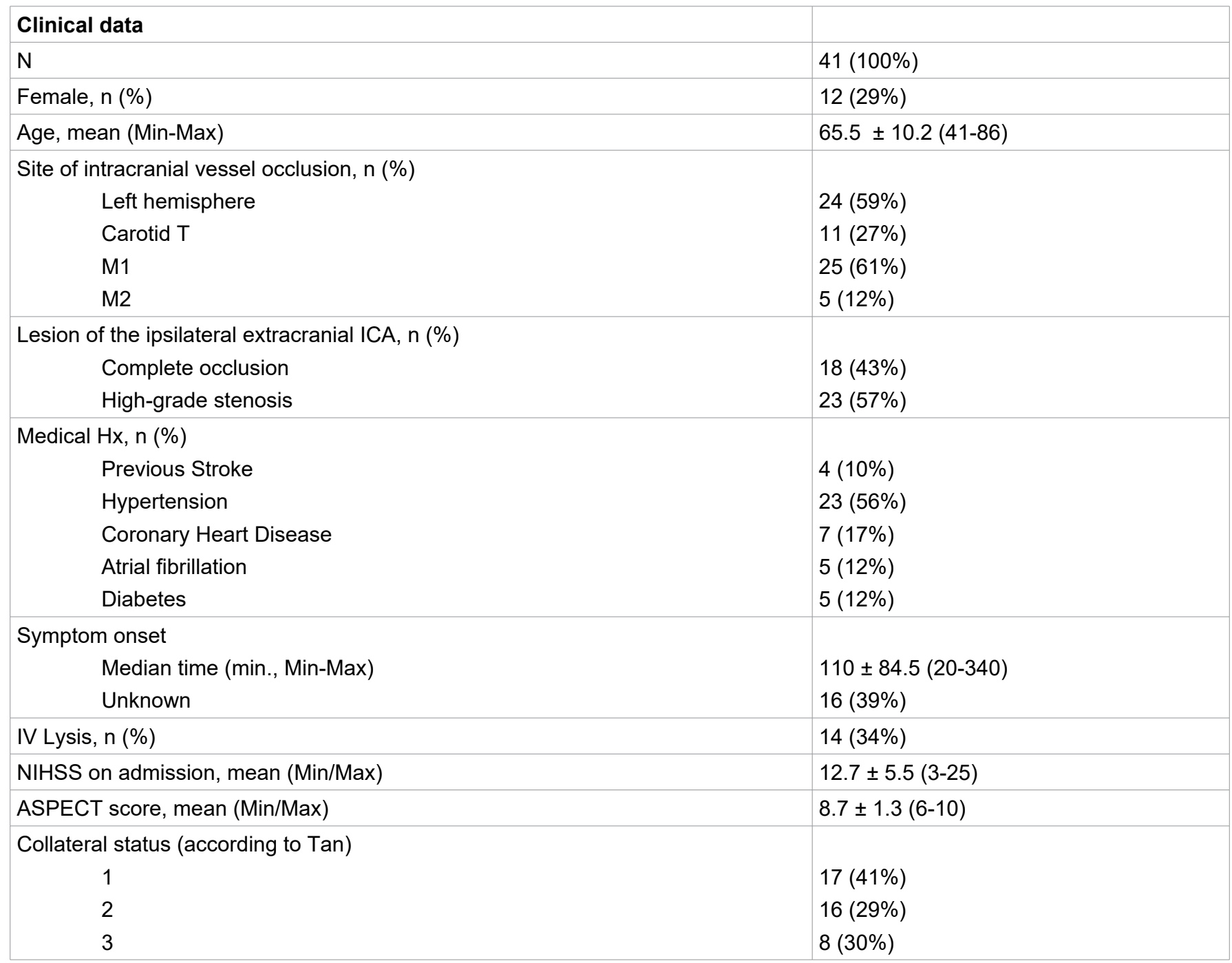

and categorical variables were tested for significance using the student's t-test and the chi-square testing, respectively. The effect of different variables on clinical outcome was tested by [1] a dichotomised approach comparing the proportion of patients with an $\mathrm{mRS} \leq 2$ versus $m R S \geq 3$, and [2] a multivariate analysis using a machine learning algorithm approach (random forest) with the statistical software " $\mathrm{R}$ ", version 3.4.2 (https:// cran.r-project.org/bin/ windows/base/old/3.4.2/). The Random Forest is a machine learning algorithm that creates thousands of decision trees, giving an estimate of what variables are important in the classification. It is an effective method for estimating missing data and maintains accuracy when a large proportion of the data are missing. A p-value of 0.05 was considered as statistically significant.

\section{Results}

From January 2012 to February 2018, 41 patients with tandem lesions in the anterior circulation were identified that matched the inclusion criteria of this study. Gender, age and clinical data of the patient cohort are given in Table 2. Recanalization of extracranial ICA and thrombectomy of the occluded vessel segment was possible in all cases. Overall, the mean number of thrombectomy manoeuvres was 2.0 [1-7], the mean time from groin puncture to the end of the procedure was $123 \pm 44 \mathrm{~min}$. (59-242). Antegrade stenting of the extracranial ICA was performed in 19 patients (46\%).

\section{Clinical outcome}

NIHSS at discharge was $9.8 \pm 12$ (0-42). Regarding the primary endpoint, mRS @90 days was available in 38 patients (92.6\%). A good clinical outcome (mRS $\leq 2$ ) was documented in 25 of 38 patients $(65.7 \%)$. There were three major bleedings and one new pontine infarction after the intervention, resulting in a complication rate of $10 \%(4 / 41)$. Overall, the mortality rate was $17 \%$ (7/41).

In multivariate testing, a favourable clinical outcome $(\mathrm{mRS} \leq 2)$ correlated with a younger age $(<70$ years; $p$ $=0.010)$, a more peripheral intracranial vessel occlusion ( $\mathrm{M} 1 / \mathrm{M} 2$ versus cartid-T; $\mathrm{p}=0.008$ ), and good cerebral collaterals ( $\operatorname{Tan}>2, p=0.012$; Table 3 and Figure 1 ). Interventional factors had no statistical effect on clinical outcome. However, there was a tendency toward a more favourable outcome in patients that underwent antegrade stenting.

\section{Discussion}

The aim of this study was to investigate the influ- 
Table 3: Effect of dichotomized clinical and interventional factors on favourable clinical outcome ( $\mathrm{mRS} \leq 2$ at 90 day follow-up).

\begin{tabular}{|c|c|c|}
\hline Clinical factors & Proportion of patients with $\mathrm{mRS} \leq 2 @ 90$ days & $p$ \\
\hline $\begin{array}{l}\text { Age } \\
<70 y \\
\geq 70 y\end{array}$ & $\begin{array}{l}19 / 23(82 \%) \\
6 / 15(40 \%)\end{array}$ & 0.007 \\
\hline $\begin{array}{l}\text { Site of intracranial vessel occlusion } \\
\text { Carotid T } \\
\text { M1/M2 }\end{array}$ & $\begin{array}{l}3 / 10(30 \%) \\
22 / 28(78.6 \%)\end{array}$ & 0.005 \\
\hline $\begin{array}{l}\text { IV Lysis } \\
\text { Yes } \\
\text { No }\end{array}$ & $\begin{array}{l}10 / 12(83.3 \%) \\
15 / 26(57.7 \%)\end{array}$ & 0.12 (n.s.) \\
\hline $\begin{array}{l}\text { NIHSS on admission } \\
<9 \\
\geq 9\end{array}$ & $\begin{array}{l}9 / 11(81.8 \%) \\
16 / 27(61.5 \%)\end{array}$ & 0.18 (n.s.) \\
\hline $\begin{array}{l}\text { ASPECT score } \\
6-8 \\
9-10\end{array}$ & $\begin{array}{l}11 / 17(64.7 \%) \\
14 / 20(70 \%)\end{array}$ & 0.56 (n.s.) \\
\hline $\begin{array}{l}\text { Collateral status (according to Tan) } \\
\text { Tan } 1 \\
\text { Tan 2-3 }\end{array}$ & $\begin{array}{l}\text { 7/15 (46.7\%) } \\
18 / 23(78.3 \%)\end{array}$ & 0.045 \\
\hline Interventional factors & & \\
\hline $\begin{array}{l}\text { Stenting } \\
\text { Antegrade } \\
\text { Retrograde }\end{array}$ & $\begin{array}{l}12 / 16(75 \%) \\
13 / 21(62 \%)\end{array}$ & 0.26 (n.s.) \\
\hline $\begin{array}{l}\text { Procedural time } \\
<60 \mathrm{~min} . \\
60 \mathrm{~min} .\end{array}$ & $\begin{array}{l}7 / 10(70 \%) \\
18 / 29(62 \%)\end{array}$ & 0.65 (n.s.) \\
\hline $\begin{array}{l}\text { No. of thrombectomy manoeuvres } \\
\leq 2 \\
\geq 3\end{array}$ & $\begin{array}{l}19 / 26(73 \%) \\
6 / 12(50 \%)\end{array}$ & 0.16 (n.s.) \\
\hline $\begin{array}{l}\text { Successful recanalization } \\
\qquad \begin{array}{l}\text { Yes }(\mathrm{TICl} 2 \mathrm{~b}-3) \\
\text { No }(\mathrm{TICl} 0-2 \mathrm{a})\end{array}\end{array}$ & $\begin{array}{l}25 / 38(56.7 \%) \\
--\end{array}$ & Not applicable \\
\hline
\end{tabular}

ence of different clinical and interventional factors on the outcome of stroke patients that underwent endovascular treatment of tandem lesions. A favourable clinical outcome at follow-up correlated significantly with a younger age, a more peripheral intracranial vessel occlusion, and good intracranial collaterals. The latter feature is known to play a crucial role in stroke treatment in general, as the existence or absence of intracranial collaterals does directly influence the size of the infarct core, the tissue at risk (penumbra), and a potential time window until successful recanalization [10-13]. It is therefore not surprising, that the grade of intracranial collateralisation had an impact on clinical outcome in our patients, as the treatment is more complex and often more time consuming than in "simple" thrombectomy. Good collaterals extend the time window for successful recanalization and might influence indirectly the grade of the neurological deficit.

Similar to the results of Park, et al. [14], we found that patients with a high-grade stenosis had a trend for a better outcome than those with complete occlusion of the extracranial ICA, however, this finding did not reach statistical significance $(p=0.052)$. Other clinical features, such as the initial NIHSS, the ASPECT-score on admission, or the affected hemisphere, did not influence long-term outcome (Figure 1).

Interestingly, we did not found interventional factors to have a major effect on clinical outcome in our cohort, either. There was a trend for a better outcome in patients that underwent antegrade stenting in comparison to retrograde stenting, however, the factor did not reach statistical significance. Other interventional factors, like the number of thrombectomy manoeuvres, the procedural time or the type of stent, had no statistical effect. An explanation for this finding might be the high proportion of technical success and the relatively high fraction of the patients with a favourable clinical outcome in our study that did not allow for a more technical in-depth-analysis in a rather small population. However, from a more clinical point of view, our analy- 


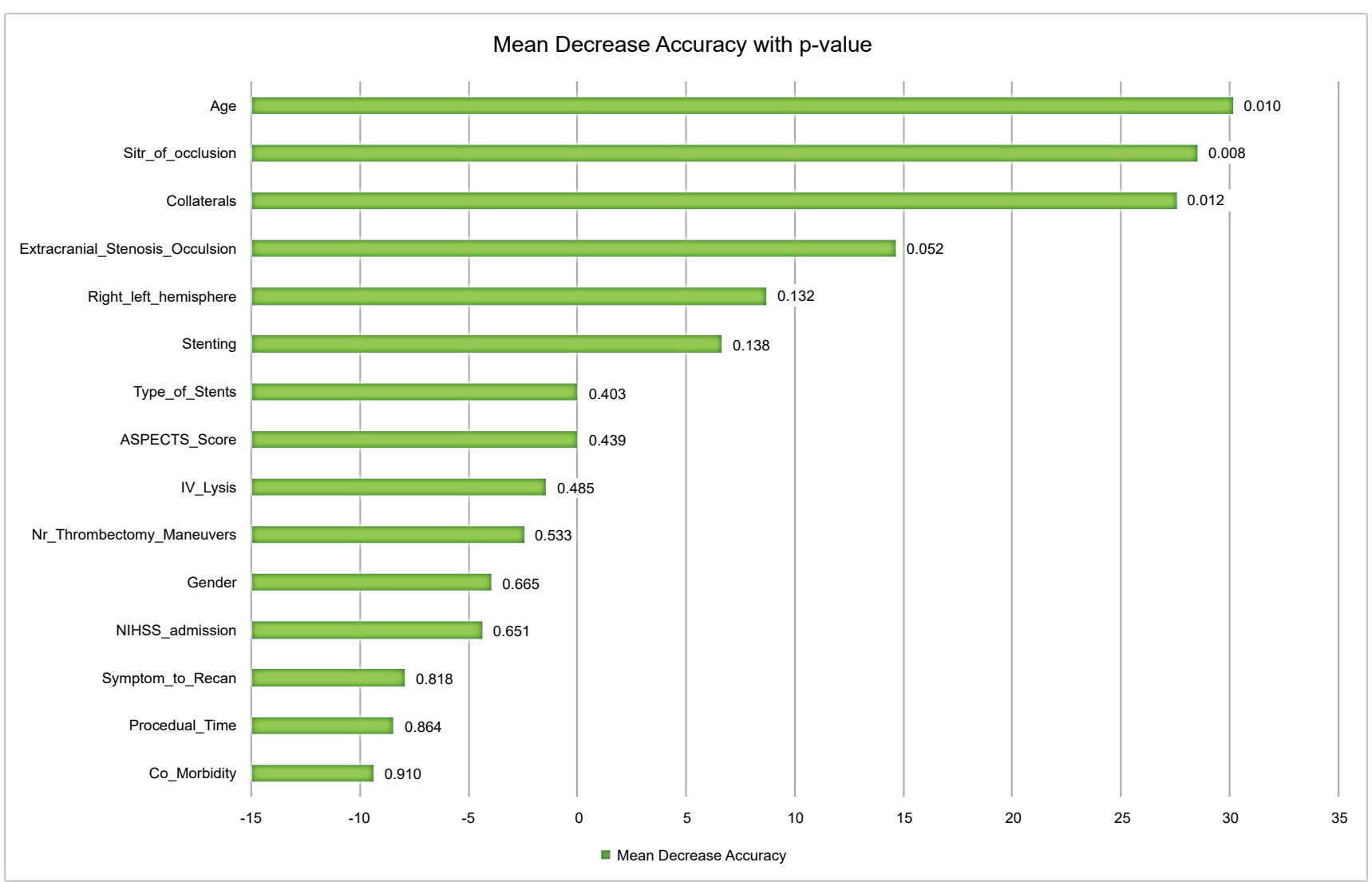

Figure 1: Impact of clinical and interventional factors on good clinical outcome at 90 -days-follow-up $(\mathrm{mRS} \leq 2)$ in 41 patients that underwent acute stroke treatment with tandem lesions. The graph shows the mean decrease in model accuracy for each feature; the corresponding p-value is given behind each bar.

sis provides evidence that patients with good intracranial collaterals and more peripheral occlusions have a high chance of a favourable outcome if they are treated successfully - independently from a specific treatment algorithm or an exact time window.

Our findings are in most parts in line with the results of other groups [15-19], however, they differ regarding the issue of antegrade versus retrograde stenting. The STRATIS collaboration group [15] demonstrated equipoise concerning antegrade vs. retrograde stenting in a prospective, non-randomized mutlicentric study with 147 patients. In contrast, Maus, et al. analysed the longterm outcome of one hundred patients after endovascular treatment of tandem lesions and found that the retrograde strategy yielded a significantly higher rate of successful reperfusion and a higher rate of favourable clinical outcome after 90 days [16]. This might be explained in parts by a faster recanalization of the intracranial vessel occlusion, when the extracranial stenosis or occlusion of the ICA is initially treated by PTA only. Moreover, a retrograde stenting approach allows for a more individual and careful decision making with regard to antiplatelet treatment in the acute setting, probably leading to a lower rate of harmful post-interventional bleedings [17]. For example, using the retrograde approach, the physician can decide not to stent immediately a moderate extracranial stenosis in a patient with unknown or long lasting symptom onset, when there is no relevant impact on the flow. Or he can decide to modify the antiplatelet regimen after stenting according to the individual situation (e.g. monotherapy with ASS or Gp2b3a-Antagonist). This is much more difficult when using the antegrade approach: Because of the limited or missing antegrade flow in the ICA before re-opening of the intracranial vessel occlusion, immediate and effective antaggregation is needed to avoid acute re-occlusion of the ICA-stent and has to be maintained, even when the intracranial recanalization is time consuming or complicated.

The major limitation of this study is its small size. The relatively low number of treated patients together with a high technical success rate influenced our statistical analysis especially with regard to interventional factors that didn't show any statistical effect on clinical outcome in our cohort as stated above. We tried to overcome these limitations by using a more progressive statistical algorithm for multivariate analysis (Random Forest), however, it is possible that some of the results that are assumed to have little or no statistical effect may not have had enough statistical power. For instance, the number of thrombectomy manoeuvres shows an estimated $23 \%$ absolute impact on good outcome proportion (Table 3): This is certainly clinically relevant, but comparisons in this range of proportion are likely to be underpowered.

Moreover, there was no core lab assessment for 
clinical and/or interventional features. This issue might have influenced especially the angiographic outcome of the interventions. We tried to overcome this limitation using a consensus reading for the assignment of the TI$\mathrm{Cl}$-score, performed by two experienced neuroradiologists (S.R., R.E.); however, this cannot completely rule out a methodical overestimation of our findings.

\section{Conclusion}

Interventional treatment of acute stroke patients with tandem lesions is technically feasible and safe. A favourable outcome can be achieved in the majority of patients and is most probable in younger patients with more peripheral intracranial occlusions and good collaterals.

\section{Funding Statement}

This research received no specific grant from any funding agency in the public, commercial or not-forprofit sectors.

\section{Competing Interests Statement}

There are no competing interests to declare.

\section{Contributorship Statement}

1. Rachid Elmouden: Design of the study, acquisition and analysis of the data, critical revision of the work, final approval of the manuscript,

2. Ana Moya: Design of the statistical analysis of the data, critical revision of the work, final approval of the manuscript,

3. Gernot Reimann: Acquisition of the data, critical revision of the work, final approval of the manuscript,

4. Olaf Adamczewski: Acquisition of the data, critical revision of the work, final approval of the manuscript,

5. Michael Schwarz: Interpretation of datacritical, revision of the work, final approval of the manuscript,

6. Stefan Rohde: Conception and design of the study, acquisition and analysis of the data, critical revision of the work, final approval of the manuscript.

\section{References}

1. Olvert A Berkhemer, Puck S S Fransen, Debbie Beumer, Lucie A van den Berg, Hester F Lingsma, et al. (2015) A randomized trial of intra arterial treatment for acute ischemic stroke. N Engl J Med 372: 11-20.

2. Bruce CV Campbell, Peter J Mitchell, Timothy J Kleinig, Helen M Dewey, Leonid Churilov, et al. (2015) Endovascular therapy for ischemic stroke with perfusion-imaging selection. N Engl J Med 372: 1009-1018.

3. Mayank Goyal, Andrew M Demchuk, Bijoy K Menon, Muneer Eesa, Jeremy L Rempel, et al. (2015) Randomized assessment of rapid endovascular treatment of ischemic stroke. N Engl J Med 372: 1019-1030.

4. Tudor G Jovin, Angel Chamorro, Erik Cobo, María A de Miquel, Carlos A Molina, et al. (2015) Thrombectomy within 8 hours after symptom onset in ischemic stroke. $\mathrm{N} \mathrm{Engl} \mathrm{J}$ Med 372: 2296-2306.
5. Jeffrey L Saver, Mayank Goyal, Alain Bonafe, Hans-Christoph Diener, Elad I Levy, et al. (2015) Stent-retriever thrombectomy after intravenous t-PA vs. t-PA alone in stroke. N Engl J Med 372: 2285-2295.

6. A J Grau, C Weimar, F Buggle, A Heinrich, M Goertler, et al. (2001) Risk factors, outcome, and treatment in subtypes of ischemic stroke: The German stroke data bank. Stroke 32: 2559-2566.

7. Wei Li, Zhonglun Chen, Zheng Dai, Rui Liu, Qin Yin, et al. (2018) Management of acute tandem occlusions: Stent-retriever thrombectomy with emergency stenting or angioplasty. J Int Med Res 46: 2578-2586.

8. Rodrigues M, Cunha A, Figueiredo S, Carvalho A, Veloso $M$, et al. (2018) Emergent carotid artery stenting in atherosclerotic disease of the internal carotid artery with tandem intracranial occlusion. J Neurol Sci 387: 196-198.

9. S Stampfl, PA Ringleb, M Möhlenbruch, C Hametner, C Herweh, et al. (2014) Emergency cervical internal carotid artery stenting incombination with intracranial thrombectomy in acutestroke. AJNR 35: 741-746.

10. Rodríguez-López C, Ballenilla Marco F, DíazGuzmán J (2018) Prognosis of Tandem Occlusions versus Isolated Intracranial Occlusions. J Stroke Cerebrovasc Dis 27: 3652-3656.

11. Rebello LC, Bouslama M, Haussen DC, Grossberg JA, Dehkharghani S (2017) Stroke etiology and collaterals: Atheroembolic strokes have greater collateral recruitment than cardioembolic strokes. Eur J Neurol 24: 762-767.

12. Jia B, Liebeskind DS, Song L, Xu X, Sun X (2017) Performance of computed tomography angiography to determine anterograde and collateral blood flow status in patients with symptomatic middle cerebral artery stenosis. Interv Neuroradiol 23: 267-273.

13. Maus V, Abdullayev N, Sack H, Borggrefe J, Mpotsaris A, et al. (2018) Carotid Artery Stenosis Contralateral to Intracranial Large Vessel Occlusion: An Independent Predictor of Unfavorable Clinical Outcome After Mechanical Thrombectomy. Front Neurol 9: 437.

14. Park SE, Choi DS, Baek HJ, Kim CH, Choi HC, et al. (2018) Endovascular therapy of acute ischemic stroke related to tandem occlusion: Comparison of occlusion and severe stenosis of the proximal cervical internal carotid artery. $\mathrm{Br} \mathrm{J}$ Radiol 92: 20180051.

15. Jadhav AP, Zaidat OO, Liebeskind DS, Yavagal DR, Haussen DC, et al. (2019) Emergent management of tandem lesions in acute ischemic stroke. Stroke 50: 428-433.

16. Maus V, Borggrefe J, Behme D, Kabbasch C, Abdullayev $\mathrm{N}$, et al. (2018) Order of Treatment Matters in Ischemic Stroke: Mechanical Thrombectomy First, Then Carotid Artery Stenting for Tandem Lesions of the Anterior Circulation. Cerebrovasc Dis 46: 59-65.

17. Díaz-Pérez J, Parrilla G, Espinosa de Rueda M, Cabrera-Maqueda JM, García-Villalba B, et al. (2018) Mechanical thrombectomy in acute stroke due to carotid occlusion: A series of 153 consecutive patients. Cerebrovasc Dis 46: 132-141.

18. Behme D, Mpotsaris A, Zeyen P, Psychogios MN, Kowoll A, et al. (2015) Emergency Stenting of the Extracranial Internal Carotid Artery in Combination with Anterior Circulation Thrombectomy in Acute Ischemic Stroke: A Retrospective Multicenter Study. Am J Neuroradiol 36: 2340-2345.

19. Steglich-Arnholm $H$, Holtmannspötter $M$, Kondziella $D$, Wagner A, Stavngaard T, et al. (2015) Thrombectomy assisted by carotid stenting in acute ischemic stroke management: Benefits and harms. J Neurol 262: 2668-2675. 